\title{
Incorporating Capacitance into Partial Core Transformer Models to Determine First Natural Resonant Frequencies
}

\author{
M.C. Liew \\ P.S. Bodger \\ Department of Electrical and Electronic Engineering, \\ University of Canterbury, Private Bag 4800, \\ Christchurch, New Zealand
}

Keywords: Transformer, modelling, partial core, capacitance, resonance 


\begin{abstract}
Capacitive components are added to partial core transformer models to allow resonant analysis at harmonic frequencies $(50-5 \mathrm{kHz})$. The harmonic frequency responses of partial core transformers with relatively low turns ratio are analysed with the model. Capacitive loads of various magnitudes are then connected to the secondary terminals in order to calculate loaded resonant frequencies. The calculated values are validated with test results. The harmonic frequency responses of two high voltage partial pore transformers with large turns ratio are presented. Calculations of the transformers' first natural resonant frequency were validated with test results. Additionally, a capacitive load was placed across each of the secondary terminals and the loaded resonant frequencies calculated by the model are compared to the measured values.
\end{abstract}




\section{Introduction}

A reverse design transformer equivalent circuit model has been introduced [1], which derives circuit components from the characteristics and dimensions actually used to build transformers. From the equivalent circuit, the transformer performance can be determined. This is essentially the opposite of the conventional design approach, hence the name reverse design approach. This approach has shown improved accuracy in predicting performance as compared to conventional transformer modelling [2].

This reverse design approach has since been applied to partial core transformers [3], where the return yokes and limbs of a full core transformer have been removed. Partial core transformers are being studied because the size of their core can be dramatically reduced albeit by an increase in winding turns. The combination gives better magnetisation than a coreless transformer and maintains the leakage flux at an acceptably low level. The combination also means that the overall weight of the partial core units is significantly reduced, and they are easier to manufacture than the conventional units. The calculated and measured operational performances of these transformers at normal operating temperatures have shown good agreement [3]. In addition, the operational performances of these transformers at liquid nitrogen 
temperatures have also shown good agreement [4].

In this paper capacitive components are added to the model to determine the first natural resonant frequencies of partial core transformers.

\section{Individual Capacitive Components}

Consideration is given to the axial view of the partial core transformer shown in Figure 1. The transformer windings are wound in the helical configuration as shown. Five capacitive components can be identified:

1. $C_{c 1}$ - capacitance between the core and the first primary layer

2. $C_{w 1}$ - self-capacitance of the primary winding

3. $C_{12}$ - interwinding capacitance

4. $C_{w 2}$ - self-capacitance of the secondary winding

5. $C_{2 T}$ - capacitance between the last secondary layer and the transformer tank

With respect to the capacitance between the outmost layer of the secondary winding and an earthed plane, the plane's orientation may be horizontal (usually just the ground plane) or vertical (in this case the transformer tank). 
In Figure 1 a tank is incorporated to act merely as an earthed plane at some uniform distance away from the transformer's outermost winding.

\subsection{Calculation of $C_{c 1}$}

The calculation of any capacitance can be done using the parallel plate theory [5]. This is strictly true only if the electric field can be considered linear between the plates. This is a reasonable approximation if the electrode surface separation dimension is small compared to the electrode dimensions. Consider two adjacent surfaces shown in Figure 2. The voltage of surface A is assumed to be varying linearly from $V_{A O}$ at the lower end to $V_{A P}$ at the top end. Similarly, the voltage of surface B varies linearly from $V_{B O}$ to $V_{B P}$. The voltage difference between the two surfaces should vary linearly along the length $l$ from $V_{O}=V_{B O}-V_{A O}$ at the bottom to $V_{P}=V_{B P}-V_{A P}$ at the top. The voltage difference at the element $\mathrm{d} x$ is

$$
\mathrm{d} V=V_{O}+\left(V_{P}-V_{O}\right) \frac{x}{l}
$$

The associated electrical energy within the element $\mathrm{d} x$ is

$$
\begin{aligned}
\mathrm{d} W & =\frac{1}{2} \mathrm{~d} C \mathrm{~d} V^{2} \\
& =\frac{1}{2} C_{l} \frac{\mathrm{d} x}{l}\left(V_{O}+\left(V_{P}-V_{O}\right) \frac{x}{l}\right)^{2}
\end{aligned}
$$


$C_{l}$ is the capacitance between the two surfaces within the elemental length $\mathrm{d} x$.

The total energy is calculated by integrating over the whole length $l$ :

$$
\begin{aligned}
\int \mathrm{d} W & =\int_{0}^{l} \frac{1}{2} C_{l} \frac{\mathrm{d} x}{l}\left(V_{O}+\left(V_{P}-V_{O}\right) \frac{x}{l}\right)^{2} \\
W & =\frac{1}{2} \frac{C_{l}}{l} \int_{0}^{l}\left\{V_{O}^{2}+\frac{2 V_{O}\left(V_{P}-V_{O}\right)}{l} x+\left(V_{P}-V_{O}\right)^{2} \frac{x^{2}}{l^{2}}\right\} \mathrm{d} x \\
& =\frac{1}{6} C_{l}\left(V_{O}^{2}+V_{O} V_{P}+V_{P}^{2}\right)
\end{aligned}
$$

If one of the surfaces represents a layer of a winding having a total voltage of $V$ across it, then the capacitance $C$ appearing across the terminals of the winding due to the distributed capacitance of that layer can be calculated by equating energies:

$$
\frac{1}{2} C V^{2}=\frac{C_{l}}{6}\left(V_{O}^{2}+V_{O} V_{P}+V_{P}^{2}\right)
$$

Therefore,

$$
C=\frac{C_{l}}{3 V^{2}}\left(V_{O}^{2}+V_{O} V_{P}+V_{P}^{2}\right)
$$

A primary winding with $L y_{1}$ layers, is shown in Figure 3(a). If $C_{m}$ is the parallel-plate capacitance between the first layer and the core, and $C_{l}$ is the capacitance between layers, then the primary winding equivalent capacitance 
is

$$
\begin{aligned}
C_{p} & =\underbrace{\frac{C_{m}}{3 V_{1}^{2}}\left(\frac{V_{1}}{L y_{1}}\right)^{2}}_{\text {layer } 1}+\underbrace{\frac{C_{l}}{3 V_{1}^{2}}\left(\frac{2 V_{1}}{L y_{1}}\right)^{2}}_{\text {layer } 2}+\cdots+\underbrace{\frac{C_{l}}{3 V_{1}^{2}}\left(\frac{2 V_{1}}{L y_{1}}\right)^{2}}_{\text {layer } L y_{1}} \\
& =\frac{C_{m}}{3 L y_{1}^{2}}+\frac{4 C_{l}}{3 L y_{1}^{2}}\left(L y_{1}-1\right)
\end{aligned}
$$

Therefore, the capacitance between the core and the first layer of the primary winding is

$$
C_{c 1}=\frac{C_{m}}{3 L y_{1}^{2}}
$$

To find $C_{m}$, consider the enlarged portion of the transformer showing the core, the first two layers of the primary winding, and the space between the core and the winding, as depicted in Figure $3(\mathrm{~b}) . C_{m}$ is a series of capacitances caused by different insulation layers:

$$
C_{m}=\frac{1}{\frac{1}{C_{I C 1}}+\frac{1}{C_{W I 1}}}
$$

where $C_{I C 1}=$ capacitance of the insulation between the core and the primary winding $I C 1$

$$
C_{W I 1}=\text { capacitance of primary winding wire insulation } w i_{1}
$$

Each of these insulation capacitances are calculated using the parallel-plate capacitance formula:

$$
C=\frac{\epsilon_{r} \epsilon_{o} A}{d}
$$




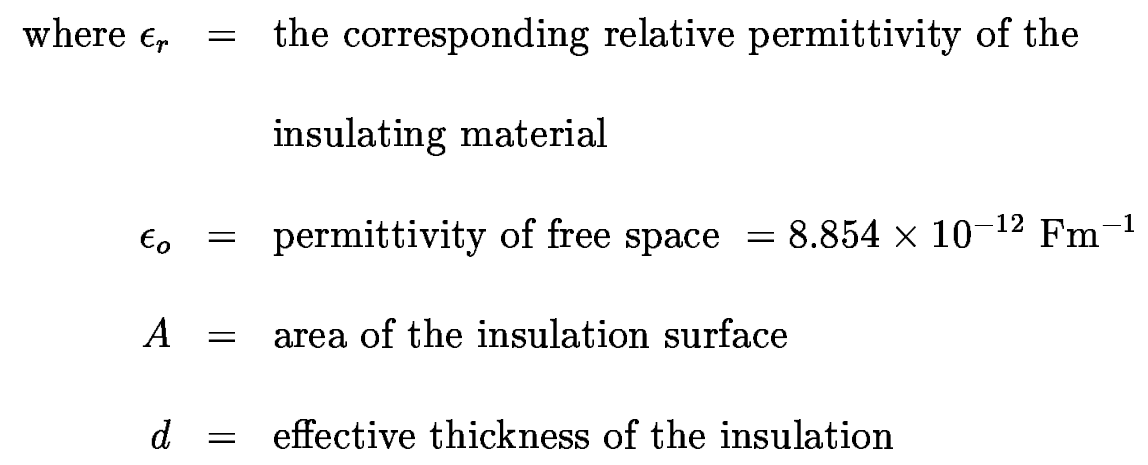

\subsection{Calculation of $C_{w 1}$}

The self-capacitance of the primary winding is calculated using the second term of Equation 6:

$$
C_{w 1}=\frac{4 C_{l}}{3 L y_{1}^{2}}\left(L y_{1}-1\right)
$$

From Figure 3(b), $C_{l}$ is calculated as

$$
C_{l}=\frac{1}{\frac{1}{C_{I 1}}+2\left(\frac{1}{C_{W I 1}}\right)}
$$

\subsection{Calculation of $C_{12}$}

The calculation of the interwinding capacitance depends on whether the number of layers in the primary winding is odd or even. Using Equation 5 and the schematics of Figures $4(\mathrm{a})$ and $4(\mathrm{~b}), C_{12}$ can be determined: 


$$
\begin{aligned}
& C_{12}= \frac{1}{3\left(V_{1}-V_{2}\right)^{2}} C_{w}\left(V_{O}^{2}+V_{O} V_{P}+V_{P}^{2}\right) \\
&=\left\{\begin{array}{lc}
\frac{1}{3\left(V_{1}-V_{2}\right)^{2}} C_{w} \times \\
{\left[\left(\frac{L y_{1}-1}{L y_{1}}\right) V_{1}\right)^{2}+\left(\frac{L y_{1}-1}{L y_{1}}\right)\left(V_{1}-\frac{V_{2}}{L y_{2}}\right) V_{1}+\left(\frac{V_{2}}{L y_{2}}-V_{1}\right)^{2}}
\end{array}\right. \\
& \begin{array}{lc}
\frac{1}{3\left(V_{1}-V_{2}\right)^{2}} C_{w} \times & \text { for } L y_{1} \text { odd } \\
{\left[\begin{array}{cc}
2 \\
\left.V_{1}^{2}+\left(\frac{L y_{1}-1}{L y_{1}}\right) V_{1}-\frac{V_{2}}{L y_{2}}\right) V_{1}+\left(\frac{V_{2}}{L y_{2}}-\left(\frac{L y_{1}-1}{L y_{1}}\right) V_{1}\right)^{2}
\end{array}\right]} & \text { for } L y_{1} \text { even }
\end{array}
\end{aligned}
$$

Examining the enlarged portion of the interwinding space between the primary and secondary windings shown in Figure 4(c), the parallel-plate capacitance $C_{w}$ between the two windings is

$$
C_{w}=\frac{1}{\frac{1}{C_{W I 1}}+\frac{1}{C_{I 12}}+\frac{1}{C_{W I 2}}}
$$

where $C_{I 12}=$ capacitance of the primary-secondary interwinding insulation $I 12$

$C_{W I 2}=$ capacitance of secondary winding wire insulation $w i_{2}$ 


\subsection{Calculation of $C_{w 2}$}

In a similar way to $C_{w 1}$, the secondary winding self-capacitance is calculated as

$$
C_{w 2}=\frac{4 C_{l}}{3 L y_{2}^{2}}\left(L y_{2}-1\right)
$$

$C_{l}$ for the secondary winding, referring to Figure $4(\mathrm{c})$, is

$$
C_{l}=\frac{1}{\frac{1}{C_{I 2}}+2\left(\frac{1}{C_{W I 2}}\right)}
$$

\subsection{Calculation of $C_{2 T}$}

To calculate the capacitance between the last layer of the secondary winding and the tank, consider the diagram of a secondary winding with $L y_{2}$ layers. Using Equation 5 and the schematic of Figure $5(\mathrm{a}), C_{2 T}$ is calculated as $[6]$

$$
\begin{aligned}
C_{2 T} & =\frac{C_{t}}{3 V_{2}^{2}}\left(V_{O}^{2}+V_{O} V_{P}+V_{P}^{2}\right) \\
& =\frac{C_{t}}{3 V_{2}^{2}}\left(V_{2}^{2}+\left(\frac{L y_{2}-1}{L y_{2}}\right) V_{2}^{2}+\left(\left(\frac{L y_{2}-1}{L y_{2}}\right) V_{2}\right)^{2}\right) \\
& =\frac{C_{t}\left(3 L y_{2}\left(L y_{2}-1\right)+1\right)}{3 L y_{2}^{2}}
\end{aligned}
$$

Once again, an enlarged portion of the transformer showing the last two layers of the secondary winding, the tank, and the space between the last layer and the tank is shown in Figure 5(b). 
The parallel-plate capacitance of $C_{t}$ is thus

$$
\begin{aligned}
& C_{t}=\frac{1}{\frac{1}{C_{W I 2}}+\frac{1}{C_{I 2 T}}} \\
& C_{I 2 T}=\text { capacitance of the insulation between secondary winding } \\
& \text { and the tank } I 2 T
\end{aligned}
$$

\section{Equivalent Transformer Capacitive Com-}

\section{ponents}

Consideration is given to the transformer equivalent circuit of Figure 6. Current in the interwinding capacitance is obtained from

$$
\begin{aligned}
i_{12} & =\omega C_{12}\left(V_{1}-V_{2}\right) \\
& =\omega C_{12}\left(V_{1}-\frac{V_{2}^{\prime}}{a}\right) \\
& =\frac{\omega C_{12}}{a}\left(V_{1}-V_{2}^{\prime}\right)+\omega C_{12}\left(\frac{a-1}{a}\right) V_{1}
\end{aligned}
$$

where $V_{2}^{\prime}=$ secondary voltage referred to the primary

Alternatively, the interwinding capacitance current entering the secondary side may be expressed as

$$
i_{12}=\omega C_{12}\left(V_{1}-V_{2}^{\prime}\right)+\omega C_{12}\left(\frac{a-1}{a}\right) V_{2}^{\prime}
$$


When referred to the primary side, Equation 19 becomes

$$
\frac{i_{12}}{a}=\frac{\omega C_{12}}{a}\left(V_{1}-V_{2}^{\prime}\right)+\omega C_{12}\left(\frac{a-1}{a^{2}}\right) V_{2}^{\prime}
$$

From Figure 6, referring the secondary capacitance to the primary and using Equations 18 and 20 to refer the interwinding capacitance to the primary side yield the equivalent circuit shown in Figure 7.

The total primary winding capacitance is

$$
C_{1}^{\prime}=C_{1}+C_{12}\left(\frac{a-1}{a}\right)
$$

The primary winding capacitive reactance is thus

$$
X_{C 1}=\frac{1}{\omega C_{1}^{\prime}}
$$

The total secondary winding capacitance is thus

$$
C_{2}^{\prime}=\frac{C_{2}}{a^{2}}+C_{12}\left(\frac{a-1}{a^{2}}\right)
$$

and the secondary winding capacitive reactance is

$$
X_{C 2}=\frac{1}{\omega C_{2}^{\prime}}
$$

The referred interwinding capacitance is

$$
C_{12}^{\prime}=\frac{C_{12}}{a}
$$

and the interwinding capacitive reactance is

$$
X_{C 12}=\frac{1}{\omega C_{12}^{\prime}}
$$




\section{Verification of the Components}

With the incorporation of the capacitive components into the reverse design partial core model, it has become possible to carry out harmonic frequency analysis of the partial core transformers. Three partial core transformers were used to compare the harmonic frequency response results between model calculations and experiments. The equivalent circuit parameters of the transformers calculated by the model are summarised in Table 1.

Table 1: Equivalent circuit parameters of the three transformers

\begin{tabular}{|c|c|c|c|}
\hline Circuit parameters & $\# 1$ & $\# 2$ & $\# 3$ \\
\hline $\begin{array}{c}\text { Resistance }(\Omega) \\
R_{\text {core }}\end{array}$ & 223.08 & 680.47 & 613.68 \\
\hline$R_{1}$ & 1.03 & 1.18 & 0.70 \\
\hline$R_{2}^{\prime}$ & 2.26 & 2.84 & 1.32 \\
\hline \hline$=\overline{=}$ & & & \\
\hline Inductance $(\mathrm{mH})$ & & & \\
$L_{m}$ & 76.66 & 116.91 & 69.47 \\
\hline$L_{1}$ & 11.37 & 7.08 & 7.95 \\
\hline$L_{2}^{\prime}$ & 11.37 & 7.08 & 7.95 \\
\hline \hline Capacitance $\overline{(\mathrm{nF})}$ & & & $=$ \\
$C_{1}^{\prime}$ & 5.81 & 5.79 & 5.86 \\
\hline$C_{2}^{\prime}$ & 0.97 & 1.04 & 1.41 \\
\hline$C_{12}^{\prime}$ & 1.02 & 1.15 & 1.81 \\
\hline
\end{tabular}

In order to confirm the validity of the model developed for frequencies up to $5 \mathrm{kHz}$, capacitive loads were connected across the secondary terminals to force the resonant frequencies of the transformers down to within power system harmonic frequency ranges. The experimental circuit is shown in Figure 8. 
The tests were conducted at three different capacitive loads for the transformers, to force resonant frequencies at $1 \mathrm{kHz}, 2 \mathrm{kHz}$ and $5 \mathrm{kHz}$ respectively. Table 2 shows the capacitive load $C_{L}$ values used for the transformers to give the three frequencies.

Table 2: $C_{L}$ values to give specified frequencies

\begin{tabular}{|c|c|c|c|}
\hline Frequency $(\mathrm{kHz})$ & $\# 1 C_{L}(\mu \mathrm{F})$ & $\# 2 C_{L}(\mu \mathrm{F})$ & $\# 3 C_{L}(\mu \mathrm{F})$ \\
\hline 1 & 36 & 48 & 19 \\
\hline 2 & 10 & 13 & 5 \\
\hline 5 & 1.9 & 2.3 & 0.9 \\
\hline
\end{tabular}

Together with the component values in Table 1 , each of the $C_{L}$ values determined from the tests was then entered into a circuit simulation package to determine the corresponding calculated resonant frequency. Table 3 shows the resonant frequencies calculated.

Table 3: Calculated frequencies

\begin{tabular}{|c|c|c|c|}
\hline $\begin{array}{c}\text { Measured frequency } \\
(\mathrm{kHz})\end{array}$ & \multicolumn{3}{|c|}{ Calculated frequency $(\mathrm{kHz})$} \\
\cline { 2 - 4 } & $\# 1$ & $\# 2$ & $\# 3$ \\
\hline 1 & 1.05 & 1.04 & 1.05 \\
\hline 2 & 2.02 & 2.00 & 2.05 \\
\hline 5 & 5.00 & 4.70 & 4.77 \\
\hline
\end{tabular}

From Table 3 it is evident that the calculated frequencies are very accurate. However, having verified the harmonic model by putting a capacitive load on the secondary winding does not necessarily imply that the inherent capacitive components of the transformer developed are correct. This is because the magnitude of the transformers' inherent capacitances are much 
smaller than the capacitive loads $C_{L}$ 's. The accuracy of the results obtained above most confirms the validity of the inductive reactance components, especially the leakage reactance. This is because the leakage reactance usually determines the transformer's first natural resonant frequency [7]. It is therefore necessary to check the accuracy of the capacitive components developed against experimental results on transformers which have significant internal capacitance.

From Equation 23, it can be seen that if a transformer with a very high secondary to primary turns ratio is designed, the transformer's turns ratio, $a$, becomes a very small value. As a result, the secondary winding capacitance, when referred to the primary, becomes significantly large. This affects and therefore lowers the transformer's first natural resonant frequency [7]. To investigate the accuracy of predicting the resonant performance of high turns ratio transformers, two high voltage partial core transformers have been built. They have the specifications shown in Table 4 .

Both transformers were designed as scale models of a high voltage transformer for testing power system components at harmonic frequencies. The equivalent circuit parameters, as determined by the model, are shown in Table 5 . 
Table 4: Specifications and design data for the transformers

\begin{tabular}{|l|c|c|}
\hline Transformer & $H V P C 1$ & $H V P C 2$ \\
\hline Ratings: & 14 & 12 \\
Primary voltage (V) & 4.56 & 6 \\
\hline Secondary voltage (kV) & 620 & 1000 \\
\hline VA rating (VA) & 45 & 50 \\
\hline Number of primary turns & 14,605 & 24,853 \\
\hline Number of secondary turns & 110 & 132 \\
\hline Core: & 76 & 64 \\
Length (mm) & 6 & 6 \\
\hline Diameter (mm) & 2 & 2 \\
\hline Core/LV winding insulation thickness (mm) & 3.5 & 4.25 \\
\hline LV winding: & 0 & 0 \\
Number of layers & 0.5 & 0.8 \\
\hline Wire diameter (mm) & & \\
\hline Interlayer insulation thickness (mm) & 20 & 29 \\
\hline LV/HV winding insulation thickness (mm) & 0.11 & 0.11 \\
\hline HV winding: & 0.15 & 0.1 \\
\hline Number of layers & & \\
\hline Wire diameter (mm) & & \\
\hline Interlayer insulation thickness (mm) & & \\
\hline
\end{tabular}

Table 5: Equivalent circuit parameters

\begin{tabular}{|c|c|c|}
\hline Circuit parameters & $H V P C 1$ & $H V P C 2$ \\
\hline $\begin{array}{c}\text { Resistance }(\Omega) \\
R_{\text {core }}\end{array}$ & 44.4 & 12.8 \\
\hline$R_{1}$ & 0.024 & 0.016 \\
\hline$R_{2}^{\prime}$ & 0.088 & 0.058 \\
\hline $\begin{array}{c}\text { Inductance }(\mathrm{mH}) \\
L_{m}\end{array}$ & 0.474 & 0.487 \\
\hline$L_{1}$ & 0.014 & 0.018 \\
\hline$L_{2}^{\prime}$ & 0.014 & 0.018 \\
\hline $\begin{array}{c}\text { Capacitance }(\mu \overline{\mathrm{F}}) \\
C_{1}^{\prime}\end{array}$ & $1.675 \times 10^{-3}$ & $0.819 \times 10^{-3}$ \\
\hline$C_{2}^{\prime}$ & 47.2 & 156 \\
\hline$C_{12}^{\prime}$ & $0.272 \times 10^{-3}$ & $0.216 \times 10^{-3}$ \\
\hline
\end{tabular}


The referred secondary winding capacitances $C_{2}^{\prime}$ 's are significantly larger in magnitude than $C_{1}^{\prime}$ and $C_{12}^{\prime}$. The open circuit frequency responses of each transformer are simulated and plotted in Figures 9(a) and 10(a).

From Figure 9(a), the natural frequency of $H V P C 1$ is found to be $4.4 \mathrm{kHz}$. The measured frequency response is also plotted in Figure 9(a). The measured resonant frequency was $4.5 \mathrm{kHz}$, which matched that of the calculated value. The measured amplitude of the resonant peak is much lower than that calculated from the model is an expected outcome. This is because in all tuned filters using magnetic core inductances, the losses increase considerably near the resonant frequency. The quality factor of the circuit, hence the magnitude of the peak, is decreased [8]. This has not been modelled.

The open circuit frequency response of $H V P C 2$ was simulated and is depicted in Figure 10(a). The simulated resonant frequency is $2.1 \mathrm{kHz}$. The frequency response of $H V P C 2$ was measured, and is also plotted in Figure 10(a). The simulated and measured results again match, confirming the validity of the capacitive components developed under open circuit conditions.

A capacitive load of $1000 \mathrm{pF}$ was then connected to the secondary winding of each transformer. $1000 \mathrm{pF}$ was chosen to represent the scaled loading effect 
of a capacitive voltage transformer [9]. When referred to the primary, the load becomes

$$
\begin{aligned}
C_{L}^{\prime} & =\frac{1}{a^{2}} C_{L} \\
& =\left(\frac{14605}{45}\right)^{2} \times 1000 \mathrm{pF} \\
& =105.3 \mu \mathrm{F}
\end{aligned}
$$

for $H V P C 1$, and

$$
\begin{aligned}
C_{L}^{\prime} & =\left(\frac{24853}{50}\right)^{2} \times 1000 \mathrm{pF} \\
& =247.1 \mu \mathrm{F}
\end{aligned}
$$

for $H V P C 2$.

The frequency response of $H V P C 1$ with the capacitive load was simulated. It is shown in Figure 9(b). Again, it can be seen that the magnitude of the measured resonant peak is much lower than that of the model. The resonant frequency was estimated to be $2.4 \mathrm{kHz}$. The loaded frequency response was also measured and plotted in Figure 9(b). The measured resonant frequency was $1.9 \mathrm{kHz}$.

Finally, the frequency response of $H V P C 2$ under the loaded condition was simulated. The results are shown in Figure 10(b). The simulated frequency is approximately $1.3 \mathrm{kHz}$. The loaded frequency response was measured, and the resonant frequency was determined to be $1.1 \mathrm{kHz}$. The result is also plotted in Figure 10(b) for comparison. The simulated and measured reson- 
ant frequencies are close enough to be useful in determining the transformer bandwidths. Again, the magnitude of each of the measured resonant peaks is much lower than that of the model due to reduced quality factors of the circuits. The closeness of the calculated and measured results for the transformers validates the usefulness of the models derived in the paper.

\section{Conclusions}

Capacitive components have been added into the reverse design partial core transformer model. The harmonic frequency response of three partial core transformers with relatively low turns ratio have been analysed. Capacitive loads were connected to the secondary terminals in order to calculate loaded resonant frequencies. Acceptable matches between the calculated and the test values were achieved. Two high voltage partial core transformers with large turns ratios were designed, built and analysed. Calculations of the transformers' resonant frequencies, under both open circuit and loaded conditions, were verified by experimental results. This has strengthened the use of the reverse design partial core model developed as an entry-level design tool from which more accurate designs can be made. 


\section{References}

[1] P.S. Bodger and M.C. Liew, "Reverse as-built transformer design method", in Int. J. Elect. Enging. Educ., 2002, vol. 39/1, pp. 42-53.

[2] P S. Bodger, M C. Liew, and P T. Johnstone, "A comparison of conventional and reverse transformer design", in Australasian Universities Power Engineering Conference (AUPEC), Brisbane, Australia, September 2000 , pp. $80-85$.

[3] M.C. Liew and P.S. Bodger, "Partial core transformer design using reverse modelling techniques", in IEE Proceedings-Electric Power Applications, November 2001, vol. 148, Issue 6, pp. 513-520.

[4] M.C. Liew and P.S. Bodger, "Operating partial core transformers under liquid nitrogen conditions", in IEE Proceedings-Electric Power Applications, July 2001, vol. 148, Issue 4, pp. 293-298.

[5] E.C. Snelling, Soft Ferrite: Properties and Applications, Butterworth \& Co. Ltd, London, UK, 2nd edition, 1988.

[6] K A. Macfayden, Small Transformers and Inductors, Chapman and Hall Ltd., London, England, 1953.

[7] Q. Jiang and P.S. Bodger, "Harmonic response and terminal resonances 
of high voltage transformers", in Int. J. Elec. Engineering Educ., April 1991, vol. 28 , No. 2 , pp. $144-156$.

[8] G. Olivier, R P. Bouchard, Y. Gervais, and D Mukhedkar, "Frequency response of $\mathrm{HV}$ test transformers and the associated measurement problems", in IEEE Transactions on Power Apparatus and Systems, January 1980, vol. PAS-99, No. 1, pp. 141-145.

[9] D.A. Bradley, P.S. Bodger, and P.R. Hyland, "Harmonic response tests on voltage transducers for the New Zealand power system", in IEEE $P A S$, July 1985, vol. 104, No. 7, pp. 1750-1756. 


\section{List of Figures}

1 Axial view of the transformer showing individual capacitances

2 Two adjacent conductive surfaces, each having a linear potential distribution

3 Calculating the primary winding self-capacitance

4 Determining the interwinding capacitance $C_{12}$

$5 \quad$ Calculating $C_{2 T}$

6 Transformer equivalent circuit with capacitive components

7 Transformer equivalent circuit, with all components referred to the primary

$8 \quad$ Frequency response test circuit

$9 \quad$ Frequency response of $H V P C 1$

10 Frequency response of $H V P C 2$ 


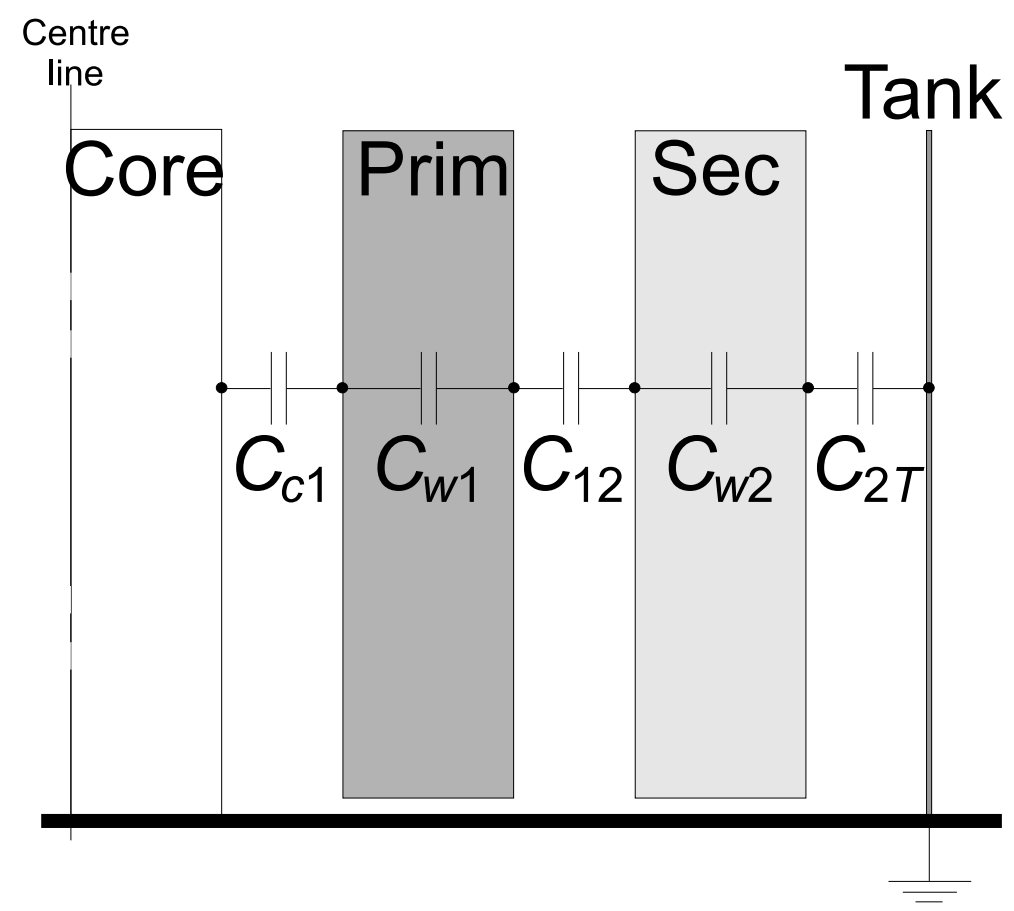

Figure 1: Axial view of the transformer showing individual capacitances 


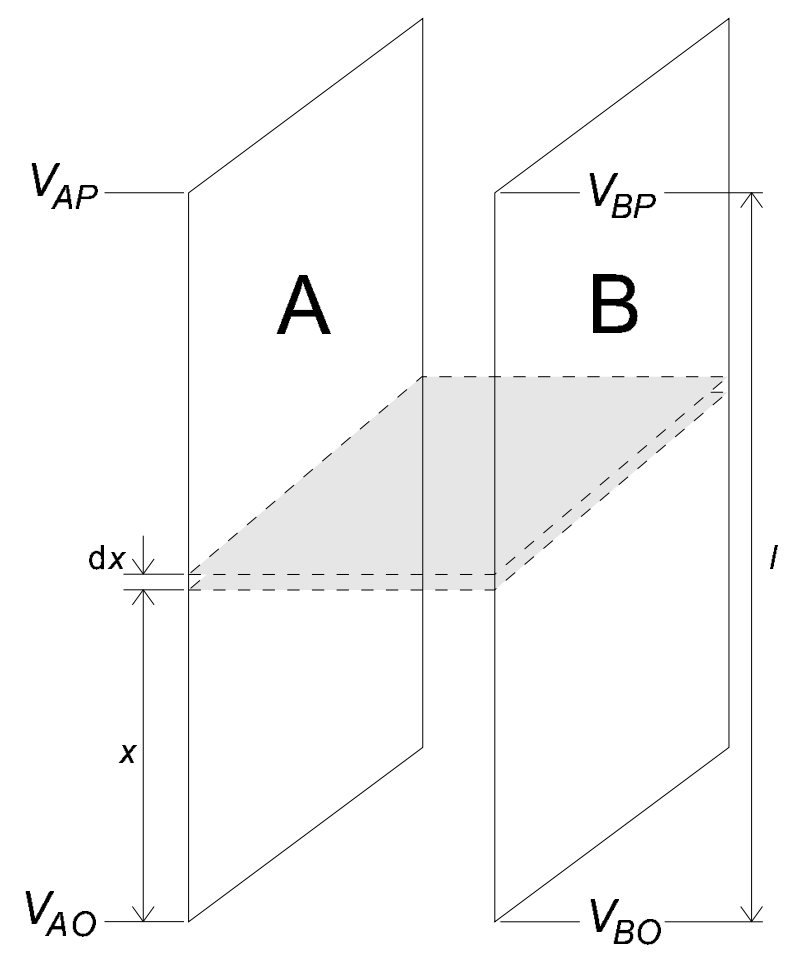

Figure 2: Two adjacent conductive surfaces, each having a linear potential distribution 


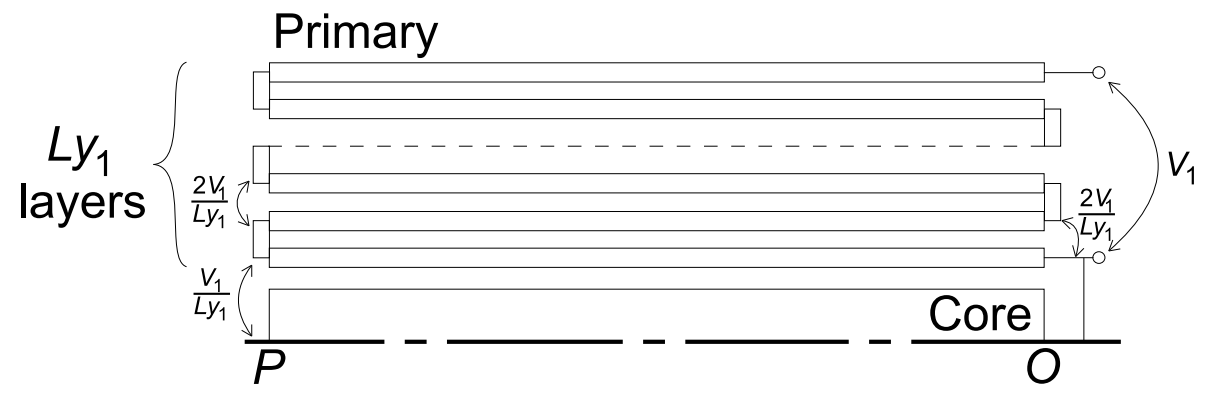

(a)

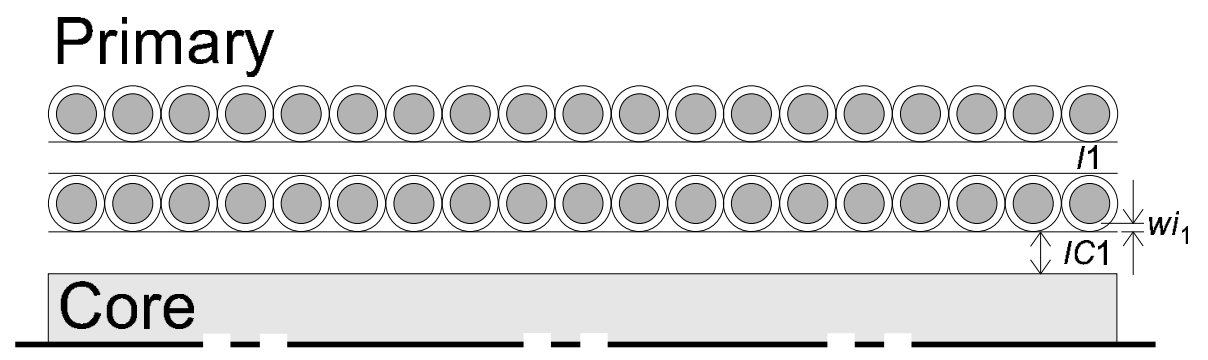

(b)

Figure 3: Calculating the primary winding self-capacitance 


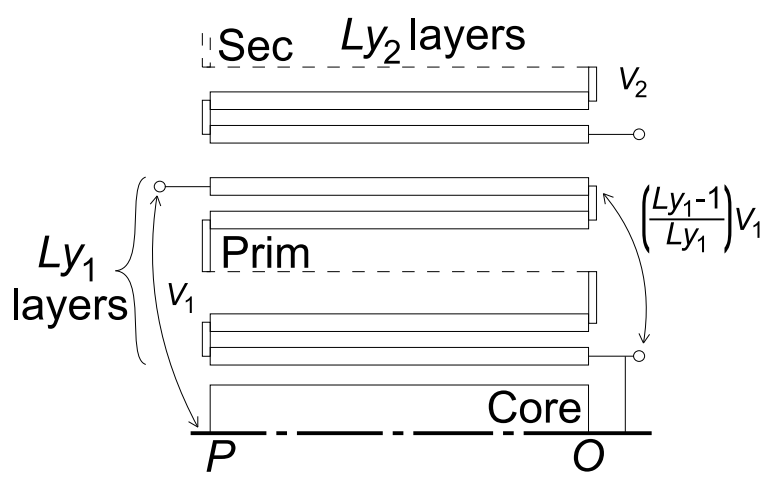

(a) $L y_{1}$ odd

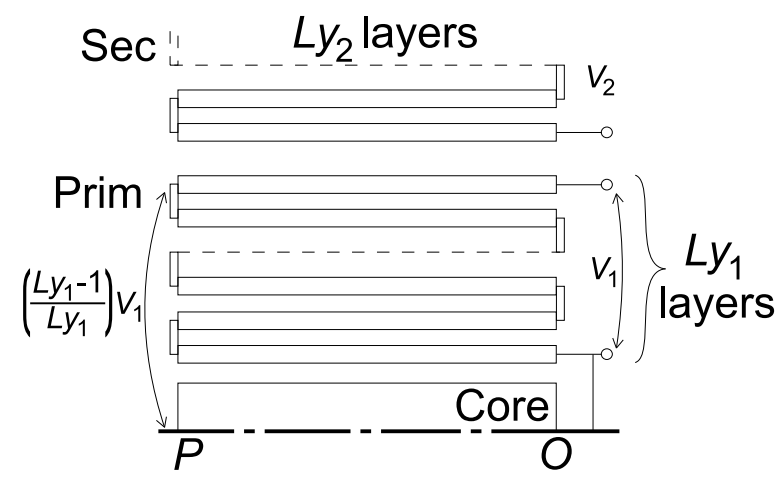

(b) $L y_{1}$ even

\section{Secondary}

0.0000000000000000000000000000012

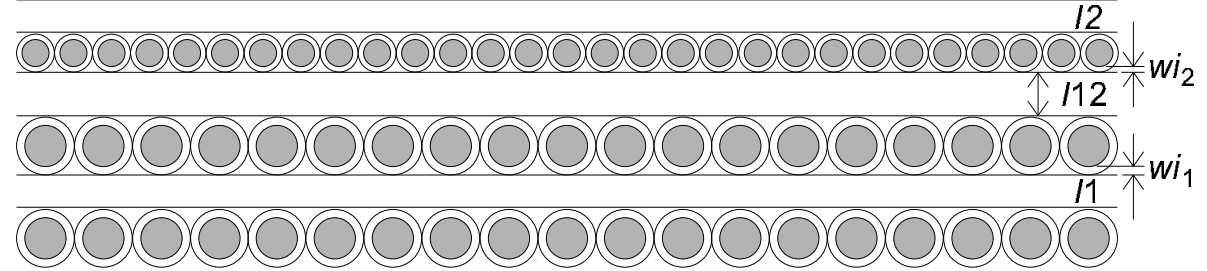

Primary

(c) Space between the primary and secondary windings

Figure 4: Determining the interwinding capacitance $C_{12}$ 


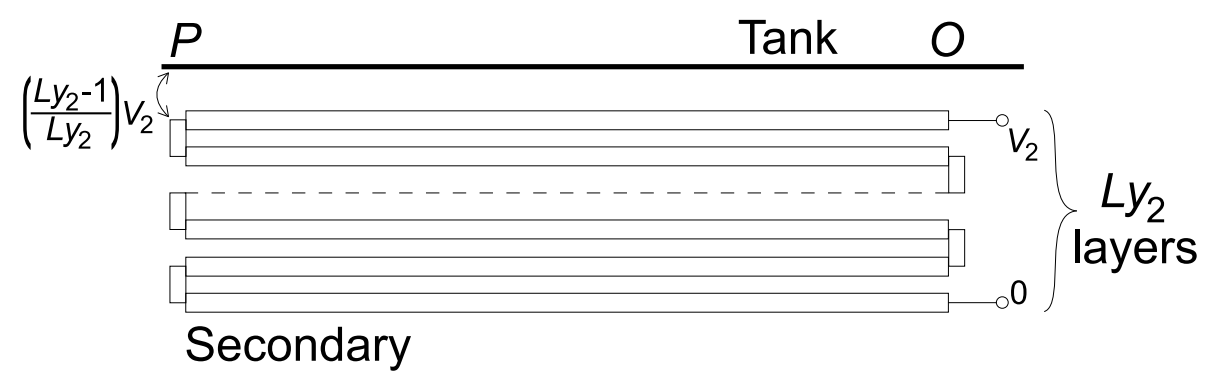

(a)

\section{Tank}

$000000000000000000000000000000 \% i_{2}$ 0000000000000000000000000000

\section{Secondary}

(b)

Figure 5: Calculating $C_{2 T}$ 


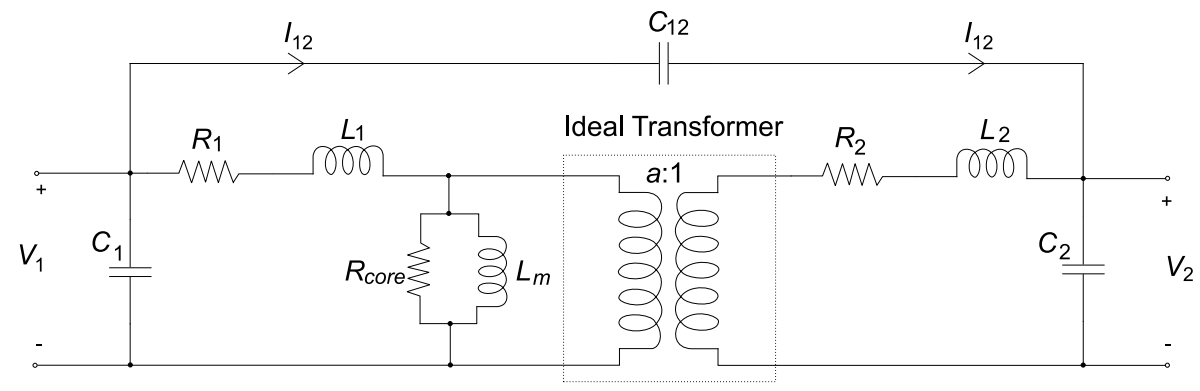

Figure 6: Transformer equivalent circuit with capacitive components 


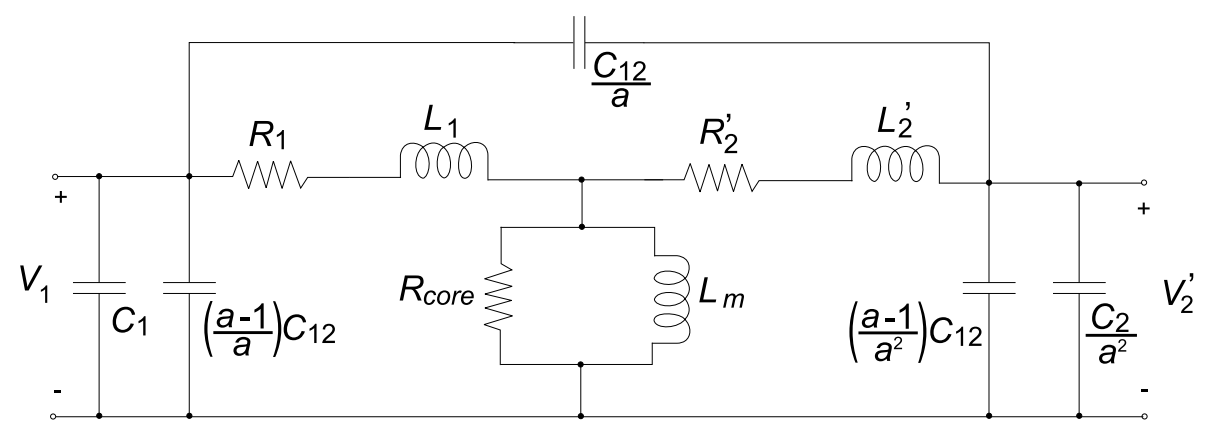

Figure 7: Transformer equivalent circuit, with all components referred to the primary 


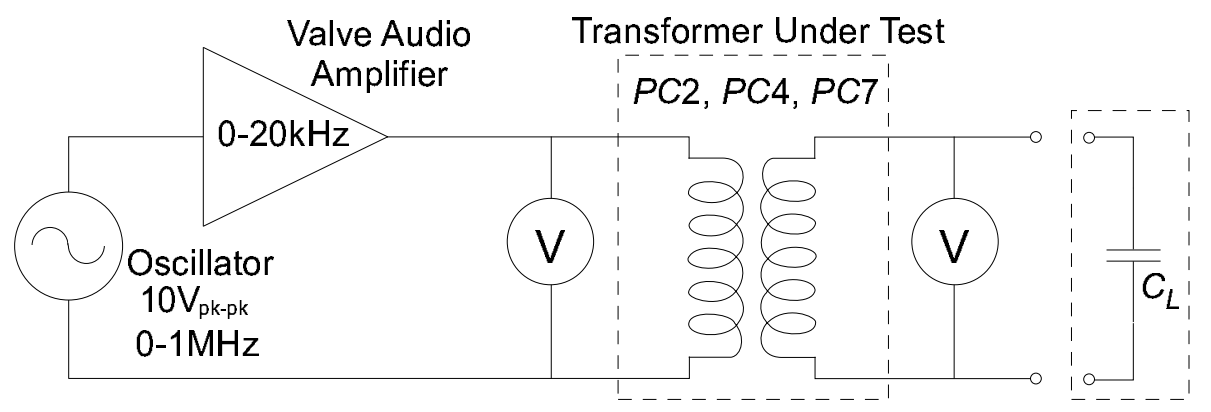

Figure 8: Frequency response test circuit 


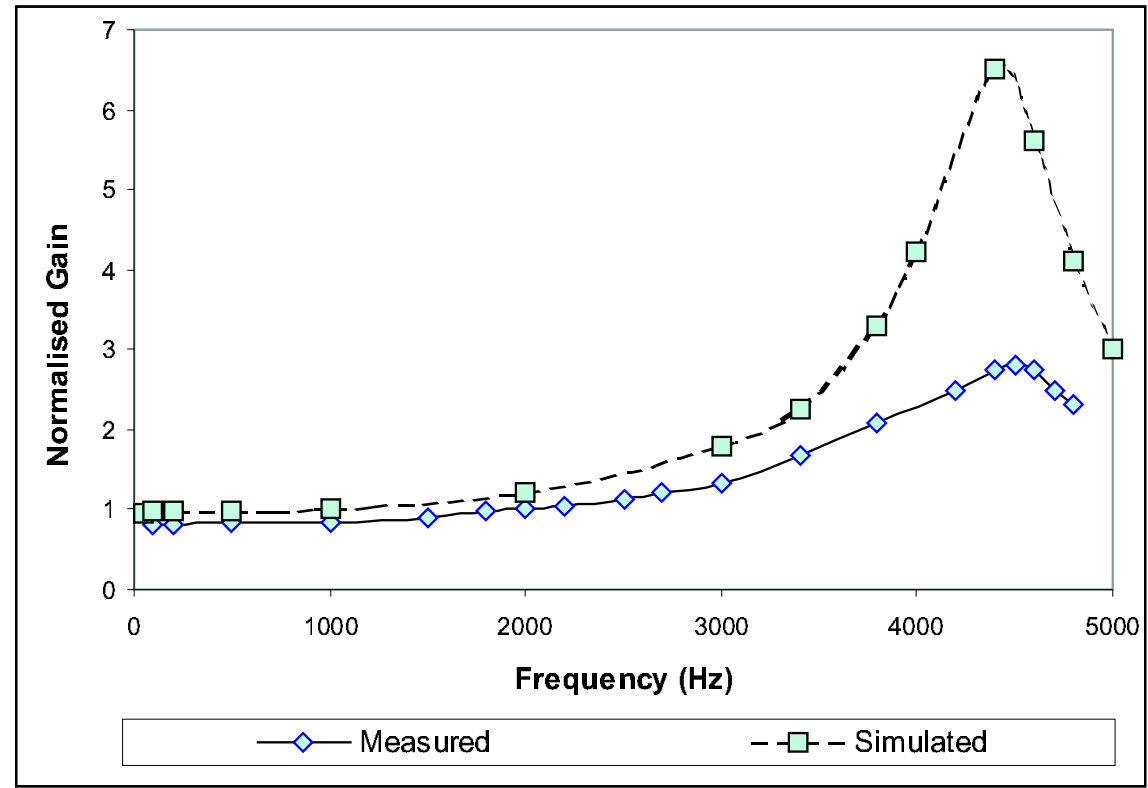

(a) Open circuit response

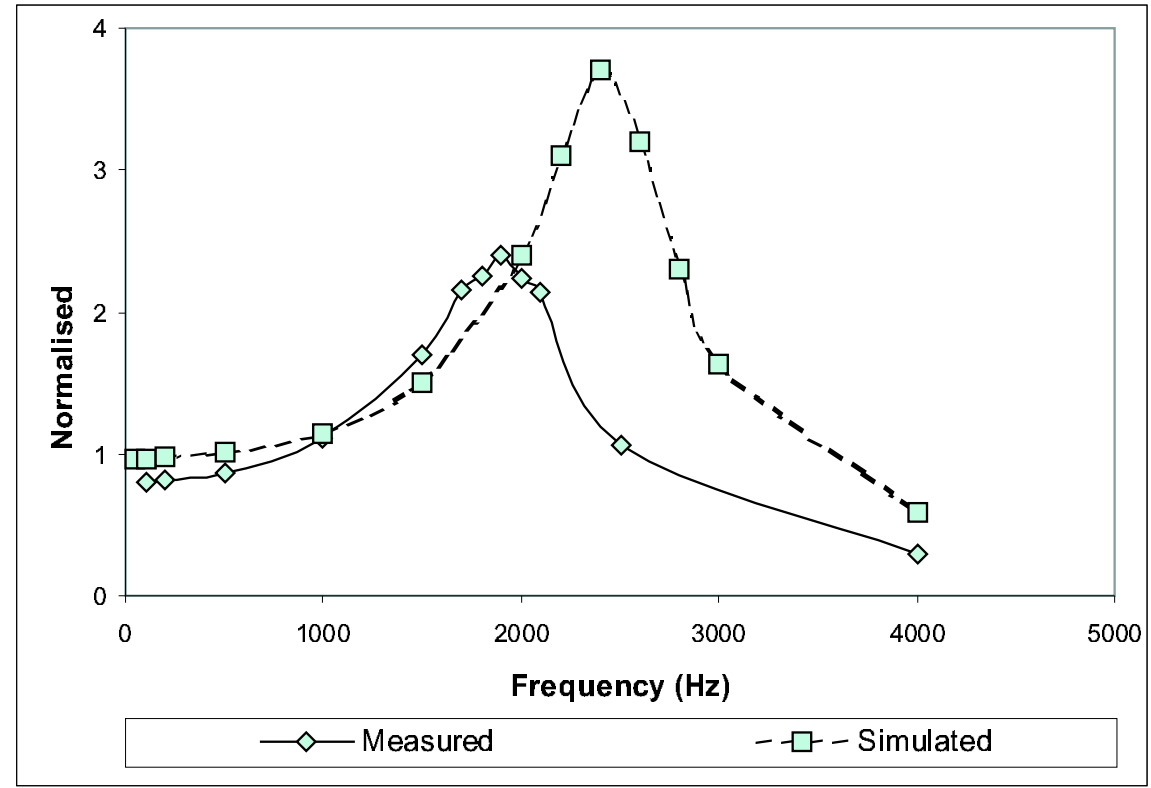

(b) Loaded response

Figure 9: Frequency response of $H V P C 1$ 


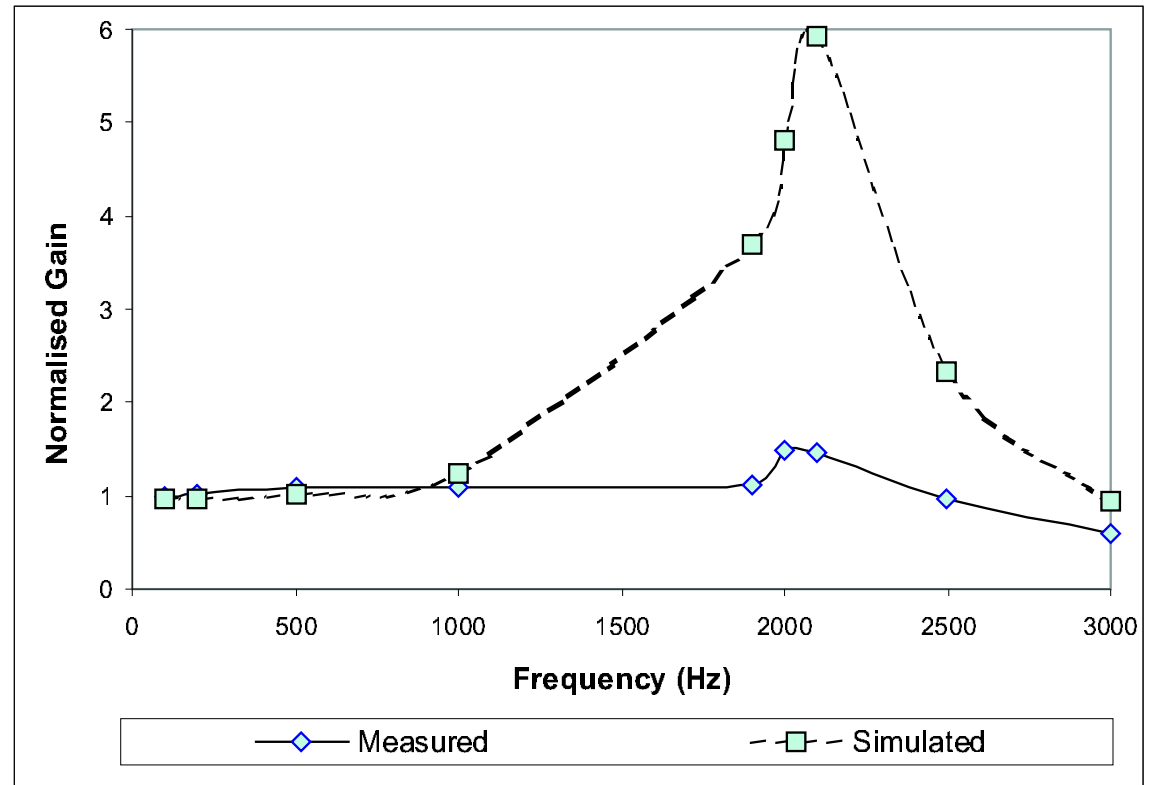

(a) Open circuit

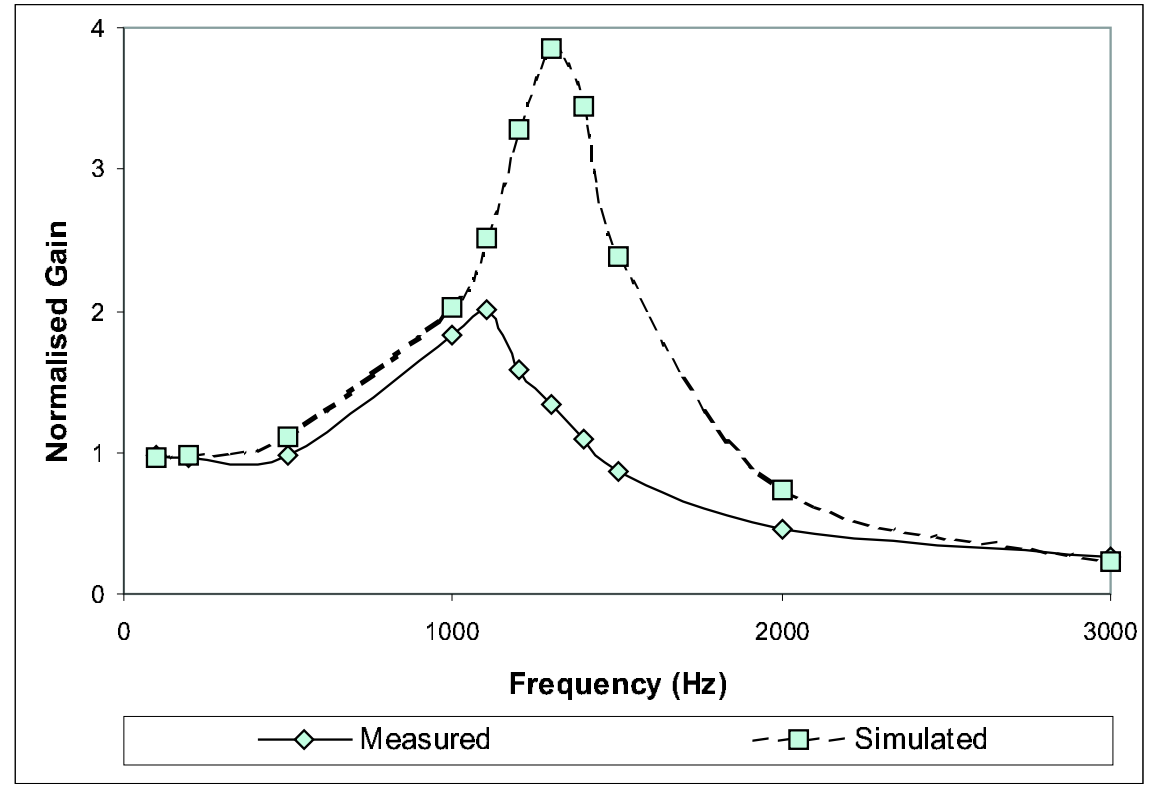

(b) Loaded response

Figure 10: Frequency response of $H V P C 2$ 